\title{
THE IMPLICIT GENERALIZED ORDER COMPLEMENTARITY PROBLEM AND LEONTIEF'S INPUT-OUTPUT MODEL
}

Abstract. We consider the Implicit Generalized Order Complementarity Problem and we use this mathematical model to study a nonlinear and conceptual generalization of Leontief's input-output economic model. We suppose that the economic system works with several technologies and the considered functions are not necessarily increasing.

Introduction. The classical Complementarity Problem is well known as an interesting and important problem in applied mathematics, which increasingly has become a cross-point between fundamental mathematics and applied mathematics [2], [12], [13]. It has been intensively studied and used as mathematical model in many practical problems in Economics, Mechanics, Elasticity, Fluid Mechanics, Engineering, Game Theory, Optimization, etc. [1], [4-6], [8], [11-16], [21], [23-25], [27], [28], [30], [32], [34].

However, in a large class of problems, the complementarity condition appears with more than one operator different from the identity operator and hence a new theoretical and numerical development is necessary.

The Implicit Complementarity Problem was the first complementarity problem with a complementarity condition defined by two operators different from the identity operator. This problem was studied by several authors [12], [13], [15] (and their references). Some interesting results were obtained, but such an approach is still unable to treat complementarity problems with the complementarity condition defined by more than two operators.

It seems that a natural way to introduce the complementarity condi-

1991 Mathematics Subject Classification: Primary 90A11.

Key words and phrases: Implicit Generalized Order Complementarity Problem, nonlinear input-output system, ordered spaces. 
tion for several operators is to use the ordering property of certain normed spaces. Proceeding in this way we obtained the Generalized Order Complementarity Problem. The first paper on this problem was [15]. In [16] we introduced the Multivalued Generalized Order Complementarity Problem and we obtained some existence results. Several practical problems from diverse scientific fields which have as a mathematical model the Generalized Order Complementary Problem are presented in [14-16].

In this paper we consider the Implicit Generalized Order Complementarity Problem and we use it to study a nonlinear and conceptual generalization of Leontief's input-output economic model.

We consider a nonlinear mathematical model for the interindustrial relations of an economical system and we are interested to know under what conditions the system is able to satisfy any demand with a minimal social cost.

The first mathematical model for the interindustrial relations was proposed by W. W. Leontief in 1949 in the classical book "The Structure of the American Economy 1919-1935", Oxford University Press, New York, 1949. Leontief's input-output model is a linear model and in some sense it is idealistic.

In 1974, A. Tamir [30] considered a nonlinear generalization of Leontief's model but supposing that the functional relations between sectors are given by increasing functions. Tamir's model was also studied by P. Bod [2], [3] using the indifferent optimization introduced by G. Wintgen [33]. The results proved by A. Tamir and P. Bod show that Tamir's nonlinear model globally works as a linear model.

The model we present in this paper is more realistic than Tamir's. The interindustrial relations between sectors are also supposed to be nonlinear functions but not necessarily increasing. We also suppose that the system is working with several technologies, in the sense that every sector is working with the same number of technologies. It is important to remark that our model is different from von Neumann's model [31] used to study the equilibrium between production and price in an expanding economy. Von Neumann's model is linear and it was studied and generalized by several authors [19], [20]. The problems studied for von Neumann's model are different from the problem studied in our paper.

Since in our model the functional relations between sectors of the system are nonlinear and not necessarily increasing an important mathematical problem is the following: under what conditions does this system have a global behavior similar to a linear system?

We solve this problem introducing the notion of tolerant economic system. To study such systems and to solve the problems defined before we will use the Implicit Generalized Order Complementarity Theory, which as 
a mathematical instrument is different than the mathematics used in the study of von Neumann's model.

In this way we will show that, if an economic system is functional and tolerant (in the sense of the definitions given in this paper), then it has the capacity to satisfy every attainable demand with a minimal social cost. Some iterative methods to approximate the optimal production are also presented. Our model can be considered as an extension to the nonlinear case of the model studied by A. Tamir [30] and P. Bod [2-3], and as an application of the Generalized Order Complementarity Problem.

1. Preliminaries. We consider the Euclidean space $\left(\mathbb{R}^{n},\langle\cdot, \cdot\rangle\right)$ endowed with the ordering defined by the pointed closed convex cone $\mathbb{R}_{+}^{n}$. We have $x \leq y$ if and only if $y-x \in \mathbb{R}_{+}^{n}$. The ordered space $\left(\mathbb{R}^{n},\langle\cdot, \cdot\rangle, \mathbb{R}_{+}^{n}\right)$ is a vector lattice, that is, for every pair $(x, y) \in \mathbb{R}^{n} \times \mathbb{R}^{n}$ the supremum $x \vee y$ and the infimum $x \wedge y$ with respect to the ordering $\leq$ exist in $\mathbb{R}^{n}$. The principal properties of supremum and infimum proved in [26] will be assumed and used here. Moreover, the vector lattice $\left(\mathbb{R}^{n}, \mathbb{R}_{+}^{n}\right)$ is a complete vector lattice, that is, $\sup (\mathbf{A})$ and $\inf (\mathbf{A})$ exist for every order bounded nonempty set $\mathbf{A} \subset \mathbb{R}^{n}$. [We say that $\mathbf{A} \subset \mathbb{R}^{n}$ is order bounded if there exist $u_{0}, v_{0} \in \mathbb{R}^{n}$ such that for every $x \in \mathbf{A}$ we have $u_{0} \leq x \leq v_{0}$.] We also remark that the cone $\mathbb{R}_{+}^{n}$ is normal and regular. To say that $\mathbb{R}_{+}^{n}$ is normal is equivalent to saying that there is a constant $\delta>0$ such that $0 \leq x \leq y$ implies $\delta\|x\| \leq\|y\|$. Since $\mathbb{R}_{+}^{n}$ is normal, every order interval in $\mathbb{R}^{n}$ is bounded. We say that $\mathbb{R}_{+}^{n}$ is regular, that is, every increasing and order bounded sequence of elements of $\mathbb{R}_{+}^{n}$ is norm convergent.

Let $\mathbf{C} \subset \mathbb{R}^{n}$ be a nonempty subset. A mapping $\mathbf{T}: \mathbf{C} \rightarrow \mathbb{R}^{n}$ is said to be isotone (resp. antitone) if for every $x_{1}, x_{2} \in \mathbf{C}$ such that $x_{1} \leq x_{2}$ we have $\mathbf{T}\left(x_{1}\right) \leq \mathbf{T}\left(x_{2}\right)$ (resp. $\mathbf{T}\left(x_{1}\right) \geq \mathbf{T}\left(x_{2}\right)$ ). If $x=\left(x_{i}\right) \in \mathbb{R}^{n}$, we write $x>0$ if $x_{i}>0$ for all $i=1, \ldots, n$.

2. The Implicit Generalized Order Complementarity Problem. We now define the Implicit Generalized Order Complementarity Problem, denoted by IGOCP, which is the mathematical instrument of this paper.

Given $m$ operators (not necessarily linear) $\mathbf{T}_{1}, \ldots, \mathbf{T}_{m}: \mathbb{R}^{n} \rightarrow \mathbb{R}^{n}$ and a nonempty subset $\mathbf{D} \subset \mathbb{R}^{n}$, the problem IGOCP associated with the family of operators $\left\{\mathbf{T}_{i}\right\}_{i=1}^{m}$ and with the set $\mathbf{D}$ is

$\operatorname{IGOCP}\left(\left\{\mathbf{T}_{i}\right\}_{i=1}^{m}, \mathbb{R}_{+}^{n}, \mathbf{D}\right):$

$$
\text { find } x_{*} \in \mathbf{D} \text { such that } \bigwedge\left(\mathbf{T}_{1}\left(x_{*}\right), \ldots, \mathbf{T}_{m}\left(x_{*}\right)\right)=0 \text {. }
$$

If $x_{*} \in \mathbf{D}$ is a solution of $\operatorname{IGOCP}\left(\left\{\mathbf{T}_{i}\right\}_{i=1}^{m}, \mathbb{R}_{+}^{n}, \mathbf{D}\right)$, then for every $i=$ $1, \ldots, m$, we have $\mathbf{T}_{i}\left(x_{*}\right) \in \mathbb{R}_{+}^{n}$. We can show that the problem 
$\operatorname{IGOCP}\left(\left\{\mathbf{T}_{i}\right\}_{i=1}^{m}, \mathbb{R}_{+}^{n}, \mathbf{D}\right)$ contains as a particular case the classical Complementarity Problem. Indeed, if $\mathbf{T}_{1}(x)=x$ for every $x \in \mathbb{R}^{n}, m=2$ and $\mathbf{D}=\mathbb{R}_{+}^{n}$, then $\mathbf{I G O C P}\left(\mathbf{I}, \mathbf{T}_{2}, \mathbb{R}_{+}^{n}, \mathbb{R}_{+}^{n}\right)$ is the problem

$\mathbf{C P}\left(\mathbf{T}_{2}, \mathbb{R}_{+}^{n}\right)$ :

find $x_{*} \in \mathbb{R}_{+}^{n}$ such that $\mathbf{T}_{2}\left(x_{*}\right) \in \mathbb{R}_{+}^{n}$ and $\left\langle x_{*}, \mathbf{T}_{2}\left(x_{*}\right)\right\rangle=0$.

When $\mathbf{D}=\mathbb{R}_{+}^{n}$, we will denote our problem by $\operatorname{IGOCP}\left(\left\{\mathbf{T}_{i}\right\}_{i=1}^{m}, \mathbb{R}_{+}^{n}\right)$. The feasible set of the problem $\mathbf{I G O C P}\left(\left\{\mathbf{T}_{i}\right\}_{i=1}^{m}, \mathbb{R}_{+}^{n}, \mathbf{D}\right)$ is by definition the set $\mathcal{F}=\left\{x \in \mathbf{D} \mid \mathbf{T}_{i}(x) \in \mathbb{R}_{+}^{n}\right.$ for $\left.i=1, \ldots, m\right\}$. We say that our problem is feasible if $\mathcal{F}$ is nonempty.

When $\mathbf{T}_{1}(x)=x$ for every $x \in \mathbb{R}^{n}$, our problem is named the Generalized Order Complementarity Problem and it is denoted by $\operatorname{GOCP}\left(\left\{\mathbf{T}_{i}\right\}_{i=2}^{m}, \mathbb{R}_{+}^{n}, \mathbf{D}\right)$.

In papers [14-16] it was shown that the problems GOCP and IGOCP have important applications. Now we formulate an IGOCP for the study of the global reproduction of an economic system working with several technologies.

3. The global reproduction of an economic system working with several technologies. Several authors used complementarity theory to study some problems in Economics [2-3], [5-6], [8], [21], [23-25], [28], [30], [32], [34].

We consider a nonlinear economic system which is a generalization of the classical linear input-output system defined by Leontief. A nonlinear generalization of this classical system with increasing functions was studied by Tamir [30] and Bod [2], [3]. A linear generalization was developed in [6] and applied to the choice of technologies. Now, we suppose that the functions are not necessarily increasing, but in some sense collectively increasing by the agency of a tolerance. Another aspect of our model is the assumption that the system is working with several technologies. For clarity, we first consider a nonlinear input-output economic system with increasing functions, but working with several technologies. We suppose that the system has $n$ production sectors and every sector works with $m$ technologies to produce one type of output. Every sector is constrained to use the production of the others. As in von Neumann's model and in all of its generalizations, we suppose that goods are produced not only from natural factors of production, but in the first place from each other. These processes of production may be circular, i.e., good $G_{i}$ is produced with the aid of $G_{j}$ and $G_{j}$ with the aid of $G_{i}$. We suppose that the number of technologies is the same for every sector.

Let $x_{j}$ be the level in units of the gross activity performed in the sector $j$. We suppose that to produce $x_{j}$ units in the sector $j, f_{i j}^{k}\left(x_{j}\right)$ units from the 
technology $k$ of the sector $i$ are needed as inputs. We make the following assumptions:

1) $f_{i j}^{k}$ are continuous,

2) $f_{i j}^{k}(0)=0$,

3) $0 \leq u_{j} \leq v_{j}$ implies $f_{i j}^{k}\left(u_{j}\right) \leq f_{i j}^{k}\left(v_{j}\right)$ for all $i, j, k$.

The balances between total activities and final demands for the technology $k$ are given by

$$
x_{i}=\sum_{j=1}^{n} f_{i j}^{k}\left(x_{j}\right)+y_{i}, \quad i=1, \ldots, n,
$$

where $y_{i}$ is the final demand for the sector $i$.

We define $f_{j}^{k}\left(x_{j}\right)=\left[f_{i j}^{k}\left(x_{j}\right)\right]_{i=1}^{n}$ for $j=1, \ldots, n$ and $\mathbf{F}^{k}(x)=x-$ $\sum_{j=1}^{n} f_{j}^{k}\left(x_{j}\right)$ for $k=1, \ldots, m$, where $x=\left(x_{1}, \ldots, x_{n}\right)^{t}$. We define

$$
\mathbf{S}_{y^{0}}=\left\{x \in \mathbb{R}_{+}^{n} \mid \mathbf{F}^{1}(x)-y^{0} \geq 0, \ldots, \mathbf{F}^{m}(x)-y^{0} \geq 0\right\} .
$$

For this model, the problem is to show that given $y^{0}>0$ with $\mathbf{S}_{y^{0}}$ nonempty the problem $\mathbf{I G O C P}\left(\mathbf{T}_{1}, \ldots, \mathbf{T}_{m}, \mathbb{R}_{+}^{n}\right)$ has a solution $x^{0}>0$ which is the least element of $\mathbf{S}_{y^{0}}$, where $\mathbf{T}_{1}(x)=\mathbf{F}^{1}(x)-y^{0}, \ldots, \mathbf{T}_{m}(x)=\mathbf{F}^{m}(x)-y^{0}$.

In this case we say that the production $x^{0}$ is realizing $y^{0}$ with a minimal social cost (in the sense of Definition 5 below). For each index $i$, there will be $m-1$ technologies which are feasible at $x^{0}$, but which create excess production. Such technologies are not thought of as active at $x^{0}$, but feasible and inactive. In case there are two or more technologies with zero excess production, for any $i$, one may choose arbitrarily among them and incur no additional cost.

Remark. If we have a final demand $y^{k}(k=1, \ldots, m)$ for every technology we can work with the final demand $y^{0}=\bigvee\left(y^{1}, \ldots, y^{m}\right)$. The model defined above is the nonlinear generalization of the classical Leontief model, but for a system working with several technologies. We denote by $\mathbf{S}_{0}\left(\left\{f_{i j}^{k}\right\}\right)$ the economic system defined by the family of mappings $\left\{f_{i j}^{k}\right\}$ satisfying assumptions 1)-3). A more realistic generalization of Leontief's system is the following. We denote by $\mathbf{I}$ the identity mapping. We now suppose that the system $\mathbf{S}_{0}\left(\left\{f_{i j}^{k}\right\}\right)$ is constrained to work with the functions $f_{i j}^{k}$ not always increasing, that is, the assumption 3 ) is not satisfied (see the economic interpretation of the functions $f_{i j}^{k}$ ). We can have the absence of assumption 3) if the system is working in the presence of strikes or other social troubles or in the presence of the import-export as considered by L. Mardon [19] or in the general model studied by A. Idzik [19]. We denote this new system by $\mathbf{S}\left(\left\{f_{i j}^{k}\right\}\right)$. The paper is dedicated to the study of this system. The following definition is essential. 
Definition 1. We say that the input-output system $\mathbf{S}\left(\left\{f_{i j}^{k}\right\}\right)$ is a tolerant economic system if the following assumptions are satisfied:

(i) $f_{i j}^{k}$ are continuous,

(ii) $f_{i j}^{k}(0)=0$,

(iii) there exists a continuous mapping $\Phi: \mathbb{R}^{n} \rightarrow \mathbb{R}^{n}$ such that:

- $\mathbf{I}+\Phi$ is invertible and $(\mathbf{I}+\Phi)^{-\mathbf{1}}$ is isotone (with respect to the ordering defined by $\mathbb{R}_{+}^{n}$ ),

- $\Phi(x)+\sum_{j=1}^{n} f_{j}^{k}\left(x_{j}\right)$ is isotone for every $k$ (where $\left.x=\left(x_{1}, \ldots, x_{n}\right)^{t}\right)$.

If $\mathbf{S}\left(\left\{f_{i j}^{k}\right\}\right)$ is a tolerant system, then we say that $\Phi$ is a tolerance.

Remarks. a) The tolerance for a given tolerant system is not unique.

b) The system $\mathbf{S}_{0}\left(\left\{f_{i j}^{k}\right\}\right)$ is tolerant with the tolerance $\Phi(x)=0$ for every $x \in \mathbb{R}^{n}$. For every tolerant system $\mathbf{S}\left(\left\{f_{i j}^{k}\right\}\right)$ consider the problem $\operatorname{IGOCP}\left(\left\{T_{k}\right\}_{k=1}^{m}, \mathbb{R}_{+}^{n}\right)$, where $T_{k}(x)=\mathbf{F}^{k}(x)-y^{0}(k=1, \ldots, m)$, associated with every final demand.

c) The concept of tolerance moves the isotonicity from the local to the global.

4. An existence theorem. Let $\mathbf{S}\left(\left\{f_{i j}^{k}\right\}\right)$ be a tolerant economic system with a tolerance $\Phi$. With the mappings $f_{j}^{k}\left(x_{j}\right)$ and $\mathbf{F}^{k}(x)$ as defined before and a final demand $y=\left[y_{i}\right]_{i=1}^{n}$ we define $\mathbf{T}_{k}(x)=\mathbf{F}^{k}(x)-y(k=1, \ldots, m)$ and $\mathbf{H}(x)=\bigvee\left\{x-\mathbf{T}_{1}(x), \ldots, x-\mathbf{T}_{m}(x)\right\}$ for all $x \in \mathbb{R}_{+}^{n}$. The mapping $\mathbf{T}_{H}(x)=(\mathbf{I}+\Phi)^{-1}(\mathbf{H}+\Phi)(x), x \in \mathbb{R}_{+}^{n}$, is well defined and it is isotone with respect to the ordering defined by $\mathbb{R}_{+}^{n}$. Let $\mathcal{F}=\left\{x \in \mathbb{R}_{+}^{n} \mid \mathbf{T}_{k}(x) \in\right.$ $\left.\mathbb{R}_{+}^{n}, k=1, \ldots, m\right\}$ be the feasible set of the system $\mathbf{S}\left(\left\{f_{i j}^{k}\right\}\right)$. We say that $\mathbf{S}\left(\left\{f_{i j}^{k}\right\}\right)$ is feasible if $\mathcal{F}$ is nonempty.

THEOREM 1. Let $\mathbf{S}\left(\left\{f_{i j}^{k}\right\}\right)$ be a tolerant economic system and let $\Phi$ be a tolerance. If $\mathbf{S}\left(\left\{f_{i j}^{k}\right\}\right)$ is feasible then the problem $\mathbf{I G O C P}\left(\left\{\mathbf{T}_{k}\right\}_{k=1}^{m}, \mathbb{R}_{+}^{n}\right)$ has a solution which is the least element of the feasible set $\mathcal{F}$.

Proof. Define $\mathbf{D}=\left\{x \in \mathbb{R}_{+}^{n} \mid \mathbf{T}_{H}(x) \leq x\right\}$. We have $\mathcal{F} \subseteq \mathbf{D}$. Indeed, if $x \in \mathcal{F}$ then $\mathbf{T}_{k}(x) \geq 0$ for all $k=1, \ldots, m$, which implies that $\left(\mathbf{I}-\mathbf{T}_{k}+\right.$ $\Phi)(x) \leq(\mathbf{I}+\Phi)(x)$ for all $k=1, \ldots, m$, that is, $\bigvee\left\{\left(\mathbf{I}-\mathbf{T}_{1}+\Phi\right)(x), \ldots,(\mathbf{I}-\right.$ $\left.\left.\mathbf{T}_{m}+\Phi\right)(x)\right\} \leq(\mathbf{I}+\Phi)(x)$, or $(\mathbf{I}+\Phi)^{-1}\left(\mathbf{T}_{H}+\Phi\right)(x) \leq x$, that is, $x \in \mathbf{D}$. We note that generally $\mathbf{D} \not \subset \mathcal{F}$. The theorem is proved if we show that $\mathbf{D}$ has a least element $x_{*}$ such that $x_{*} \in \mathcal{F}$ and $\mathbf{T}_{H}\left(x_{*}\right)=x_{*}$. Indeed, if $\mathbf{S}\left(\left\{f_{i j}^{k}\right\}\right)$ is feasible then $\mathcal{F}$ is nonempty. Since $\mathbf{D} \subseteq \mathbb{R}_{+}^{n}$ and $\left(\mathbb{R}^{n}, \mathbb{R}_{+}^{n}\right)$ is a complete vector lattice the element $x_{*}=\inf \mathbf{D}$ is well defined. For every $x \in \mathbf{D}$ we have $\mathbf{T}_{H}(x) \leq x$ and $x_{*} \leq x$, which implies that $\mathbf{T}_{H}\left(x_{*}\right) \leq \mathbf{T}_{H}(x) \leq x$ and hence $\mathbf{T}_{H}\left(x_{*}\right) \leq x_{*}$. Thus, we deduce that $x_{*} \in \mathbf{D}$. Since $\mathbf{T}_{H}\left(x_{*}\right) \leq x_{*}$ 
implies $\mathbf{T}_{H}\left(\mathbf{T}_{H}\left(x_{*}\right)\right) \leq \mathbf{T}_{H}\left(x_{*}\right)$, we see that $\mathbf{T}_{H}\left(x_{*}\right) \in \mathbf{D}$ and hence $x_{*} \leq$ $\mathbf{T}_{H}\left(x_{*}\right)$, which implies that $\mathbf{T}_{H}\left(x_{*}\right)=x_{*}$. Because $x_{*}$ is a solution of the problem IGOCP $\left(\left\{\mathbf{T}_{k}\right\}_{k=1}^{m}, \mathbb{R}_{+}^{n}\right)$ (by the definition of $\left.\mathbf{T}_{H}\right)$, it is a feasible point and hence $x_{*} \in \mathcal{F}$, which implies that $x_{*}$ is the least element of $\mathcal{F}$ (since it is the least element of $\mathbf{D}$ ).

We now introduce several definitions.

Definition 2. We say that a tolerant economic system $\mathbf{S}\left(\left\{f_{i j}^{k}\right\}\right)$ is functional if there exists $\bar{x}>0$ such that $\mathbf{F}^{k}(\bar{x})>0$ for every $k=1, \ldots, m$.

Definition 3. If $\mathbf{S}\left(\left\{f_{i j}^{k}\right\}\right)$ is a functional tolerant economic system, we say that $y^{0}>0$ is a final attainable demand if there exists $x^{0} \in \mathbb{R}_{+}^{n}$ such that $\mathbf{T}_{k}\left(x^{0}\right)=\mathbf{F}^{k}\left(x^{0}\right)-y^{0} \in \mathbb{R}_{+}^{n}$ for every $k=1, \ldots, m$.

DEFInition 4. We say that a final attainable demand $y^{0}>0$ is minimally realized by a production $x^{0} \in \mathbb{R}_{+}^{n}$ if $\mathbf{T}_{k}\left(x^{0}\right)=\mathbf{F}^{k}\left(x^{0}\right)-y^{0} \in \mathbb{R}_{+}^{n}$ for every $k=1, \ldots, m$ and $\bigwedge\left\{\mathbf{T}_{1}\left(x^{0}\right), \ldots, \mathbf{T}_{m}\left(x^{0}\right)\right\}=0$.

Definition 5. We say that a function $\varphi: \mathbb{R}_{+}^{n} \rightarrow \mathbb{R}$ is a social cost for the system $\mathbf{S}\left(\left\{f_{i j}^{k}\right\}\right)$ if:

1) $\varphi(0)=0$,

2) $\varphi(x)>0$ if $\|x\|>0$,

3) $x \leq y \Rightarrow \varphi(x) \leq \varphi(y)$.

Similar definitions were used in [30] and [4]. With the last definitions, we obtain from Theorem 1 the following interesting result.

Corollary. Let $\mathbf{S}\left(\left\{f_{i j}^{k}\right\}\right)$ be a functional tolerant economic system and let $\varphi$ be its social cost. For every attainable demand $y^{0}>0$ there exists a production $x^{0}>0$ realizing $y^{0}$ with $\varphi\left(x^{0}\right)$ minimal.

Proof. It is sufficient to remark that Theorem 1 is applicable with $\mathbf{T}_{k}(x)=\mathbf{F}^{k}(x)-y^{0}(k=1, \ldots, m)$ and the solution $x^{0}$ of the problem $\operatorname{IGOCP}\left(\left\{\mathbf{T}_{k}\right\}_{k=1}^{m}, \mathbb{R}_{+}^{n}\right)$ which is the least element of the feasible set $\mathcal{F}$ is such that $x^{0}>0$. Indeed, we have $x^{0} \in \mathbb{R}_{+}^{n}$. If we suppose that there exists $i \in\{1, \ldots, n\}$ such that $x_{i}^{0}=0$, then we have $\left[\mathbf{T}_{k}\left(x^{0}\right)\right]_{i}=x_{i}^{0}-$ $\sum_{j=1}^{n} f_{i j}^{k}\left(x_{j}^{0}\right)-y_{i}^{0}<0$, which is impossible since $x^{0}$ is a feasible point. Certainly we have $\varphi\left(x^{0}\right) \leq \varphi(x)$ for every $x \in \mathcal{F}$.

R e m a r k. Let $x^{0}>0$ be the production realizing the attainable demand $y^{0}>0$ in a functional tolerant economic system working with $m$ technologies. Since $x^{0}$ is obtained as a solution of an Implicit General Order Complementarity Problem, it can happen that the same component $y_{i}^{0}$ of $y^{0}$ is realized by several technologies $k_{1}, \ldots, k_{r}$. We may choose arbitrarily one of these without any loss of generality or change in social cost. In the next section we give some iterative methods to compute the optimal production. 
5. Iterative methods. In this section we give some iterative methods to compute the solution defined in Theorem 1 (and hence the optimal production $x^{0}$ considered in the Corollary of Theorem 1).

THEOREM 2. Let $\mathbf{S}\left(\left\{f_{i j}^{k}\right\}\right)$ be a tolerant economic system and let $\Phi$ be a tolerance. Suppose that:

1) $0 \leq \mathbf{H}(0)$,

2) $(\mathbf{I}+\Phi)^{-1}(\mathbf{H}+\Phi)$ is continuous,

3) $\mathbf{S}\left(\left\{f_{i j}^{k}\right\}\right)$ is feasible.

Then the problem $\mathbf{I G O C P}\left(\left\{\mathbf{T}_{k}\right\}_{k=1}^{m}, \mathbb{R}_{+}^{n}\right)$ has a solution $x_{*}$ which is the least element of the feasible set $\mathcal{F}$ and $x_{*}$ can be computed by the following iterations:

$$
x_{0}=0, \quad x_{r+1}=(\mathbf{I}+\Phi)^{-1}(\mathbf{H}+\Phi)\left(x_{r}\right), \quad r=0,1,2, \ldots
$$

Proof. By Theorem $1, \mathcal{F}$ (which is nonempty) has a least element $x_{*}$ which is a solution of the problem $\operatorname{IGOCP}\left(\left\{\mathbf{T}_{k}\right\}_{k=1}^{m}, \mathbb{R}_{+}^{n}\right)$. Let $y_{0} \in \mathcal{F}$ be an arbitrary element. Then $\mathbf{T}_{k}\left(y_{0}\right) \in \mathbb{R}_{+}^{m}$ for every $k=1, \ldots, m$, which implies that $-\mathbf{T}_{k}\left(y_{0}\right) \leq 0$ for every $k=1, \ldots, m$. Thus, we have $\mathbf{H}\left(y_{0}\right) \leq y_{0}$, which implies that the sequence $\left\{x_{r}\right\}_{r \in \mathbb{N}}$ defined by the algorithm $(\theta)$ has the following property: $0 \leq x_{1} \leq \ldots \leq x_{r} \leq \ldots \leq y_{0}$.

Since the cone $\mathbb{R}_{+}^{n}$ is regular there exists $\widehat{x}=\lim _{r \rightarrow \infty} x_{r}$ and because $\mathbb{R}_{+}^{n}$ is closed we have $0 \leq \widehat{x} \leq y_{0}$. By continuity we obtain $\widehat{x}=(\mathbf{I}+\Phi)^{-1}(\mathbf{H}+$ $\Phi)(\widehat{x})$, that is, $\widehat{x}$ is a solution of the problem $\operatorname{IGOCP}\left(\left\{\mathbf{T}_{k}\right\}_{k=1}^{m}, \mathbb{R}_{+}^{n}\right)$.

We now show that $\widehat{x}$ is a minimal solution of the problem $\operatorname{IGOCP}\left(\left\{\mathbf{T}_{k}\right\}_{k=1}^{m}, \mathbb{R}_{+}^{n}\right)$ with respect to the set $\left[0, y_{0}\right]=\left\{x \in \mathbb{R}^{n} \mid 0 \leq\right.$ $\left.x \leq y_{0}\right\}$. Indeed, let $z_{0}$ be an arbitrary solution of this problem in $\left[0, y_{0}\right]$. Since $z_{0}$ is a fixed point of the mapping $\mathbf{T}_{H}(x)=(\mathbf{I}+\Phi)^{-1}(\mathbf{H}+\Phi)(x)$, the sequence $\left\{x_{r}\right\}_{r \in \mathbb{N}}$ defined by the algorithm $(\theta)$ satisfies $0 \leq x_{r} \leq z_{0}$ for all $r \in \mathbb{N}$, which implies that $0 \leq \widehat{x} \leq z_{0}$, that is, $\widehat{x}$ is minimal. Because $\widehat{x}$ is in particular a feasible element we have $0 \leq x_{*} \leq \widehat{x} \leq y_{0}$, and since $\widehat{x}$ is a minimal solution in the interval $\left[0, y_{0}\right]$, we deduce that $0 \leq \widehat{x} \leq x_{*}$, that is, $x_{*}=\widehat{x}$ and the theorem is proved.

Next we compute two elements $u_{*}, v_{*}$ such that the least element solution $x_{*}$ of the problem $\operatorname{IGOCP}\left(\left\{\mathbf{T}_{k}\right\}_{k=1}^{m}, \mathbb{R}_{+}^{n}\right)$ satisfies the condition $u_{*} \leq x_{*}$ $\leq v_{*}$. Our method is based on the concept of "coupled fixed point", introduced by Lakshmikantham and studied by Guo and Lakshmikantham [10] and also on the concept of "heterotonic operator" defined by Opoitsev [22].

We say that $\mathbf{T}: \mathbb{R}^{n} \rightarrow \mathbb{R}^{n}$ is heterotonic on a set $\mathbf{D} \subset \mathbb{R}^{n}$ if and only if there exists an operator $\widehat{\mathbf{T}}: \mathbb{R}^{n} \times \mathbb{R}^{n} \rightarrow \mathbb{R}^{n}$ such that

(i) $\widehat{\mathbf{T}}(x, x)=\mathbf{T}(x)$ for all $x \in \mathbf{D}$,

(ii) $\widehat{\mathbf{T}}(x, y)$ is isotone with respect to $x$ for all $y$, 
(iii) $\widehat{\mathbf{T}}(x, y)$ is antitone with respect to $y$ for all $x$.

If $\mathbf{T}$ is heterotonic, then $\widehat{\mathbf{T}}$ is not necessarily unique. We now suppose that in the problem $\mathbf{I G O C P}\left(\left\{\mathbf{T}_{k}\right\}_{k=1}^{m}, \mathbb{R}_{+}^{n}\right)$ the operators $\mathbf{I}-\mathbf{T}_{k}$ (for every $k=1, \ldots, m)$ are heterotonic (I-T $\mathbf{T}_{k}$ heterotonic does not imply $\mathbf{T}_{k}$ isotone). Let $\widehat{\mathbf{S}}_{k}$ be the operator associated with $\mathbf{I}-\mathbf{T}_{k}$. Now consider again the mapping $\mathbf{H}(x)=\bigvee\left\{x-\mathbf{T}_{1}(x), \ldots, x-\mathbf{T}_{m}(x)\right\}$ for $x \in \mathbb{R}_{+}^{n}$, and define $\widehat{\mathbf{H}}(x, y)=\bigvee\left\{\widehat{\mathbf{S}}_{1}(x, y), \ldots, \widehat{\mathbf{S}}_{m}(x, y)\right\}$ for all $(x, y) \in \mathbb{R}_{+}^{n} \times \mathbb{R}_{+}^{n}$. Then $\widehat{\mathbf{H}}$ is isotone with respect to $x$ and antitone with respect to $y$ and moreover, $\widehat{\mathbf{H}}(x, x)=\mathbf{H}(x)$ for all $x \in \mathbb{R}_{+}^{n}$. Hence, in this case $\mathbf{H}$ is a heterotonic operator. We say that $\left(x_{*}, y_{*}\right)$ is a coupled fixed point for $\mathbf{H}$ if:

(iv) $\widehat{\mathbf{H}}\left(x_{*}, y_{*}\right)=x_{*}$,

(v) $\widehat{\mathbf{H}}\left(y_{*}, x_{*}\right)=y_{*}$.

Since $\widehat{\mathbf{H}}\left(x_{*}, x_{*}\right)=\mathbf{H}\left(x_{*}\right)$, every fixed point is a coupled fixed point but the converse is not true. A coupled fixed point $\left(x_{*}, y_{*}\right)$ of $\mathbf{H}$ is minimal and maximal on a subset $\mathbf{D} \subset \mathbb{R}^{n}$ if, for every coupled fixed point $(\bar{x}, \bar{y})$ of $\mathbf{H}$ such that $(\bar{x}, \bar{y}) \in \mathbf{D} \times \mathbf{D}$ we have $x_{*} \leq \bar{x} \leq y_{*}$ and $x_{*} \leq \bar{y} \leq y_{*}$. Given two points $u_{0}, v_{0} \in \mathbb{R}_{+}^{n}$, the conical interval $\left[u_{0}, v_{0}\right]$ is strongly invariant for $\mathbf{H}$ if:

(vi) $u_{0} \leq \widehat{\mathbf{H}}\left(u_{0}, v_{0}\right)$,

(vii) $\widehat{\mathbf{H}}\left(v_{0}, u_{0}\right) \leq v_{0}$.

Applying this concept to the current problem leads to the following result.

Theorem 3. Let $\mathbf{S}\left(\left\{f_{i j}^{k}\right\}\right)$ be a feasible tolerant economic system. If for every $k=1, \ldots, m$ the operator $\mathbf{I}-\mathbf{T}_{k}$ is heterotonic with $\widehat{\mathbf{S}}_{k}$ continuous, then for every conical interval $\left[0, v_{0}\right]$ strongly invariant for $\mathbf{H}$, there exists a minimal and maximal coupled fixed point $\left(u_{*}, v_{*}\right)$ such that $u_{*} \leq x_{*} \leq v_{*}$, where $x_{*}$ is the least element solution of the problem $\mathbf{I G O C P}\left(\left\{\mathbf{T}_{k}\right\}_{k=1}^{m}, \mathbb{R}_{+}^{n}\right)$. Moreover $u_{*}=\lim _{r \rightarrow \infty} u_{r}$ and $v_{*}=\lim _{r \rightarrow \infty} v_{r}$, where $u_{r}=\widehat{\mathbf{H}}\left(u_{r-1}, v_{r-1}\right)$ and $v_{r}=\widehat{\mathbf{H}}\left(v_{r-1}, u_{r-1}\right)$, with $u_{0}=0$.

Proof. Since in the ordered space $\left(\mathbb{R}^{n}, \mathbb{R}_{+}^{n}\right)$ the operation " $\mathrm{V}$ " is continuous, it follows from our assumptions that $\widehat{\mathbf{H}}$ is a continuous mapping. Because $\widehat{\mathbf{H}}$ is isotone with respect to $x$ and antitone with respect to $y$ and the conical interval $\left[0, v_{0}\right]$ is strongly invariant,

$$
0 \leq u_{1}=\widehat{\mathbf{H}}\left(0, v_{0}\right), \quad v_{1}=\widehat{\mathbf{H}}\left(v_{0}, 0\right) \leq v_{0} \quad \text { and } \quad 0 \leq u_{1} \leq v_{1} \leq v_{0} .
$$

By induction $0 \leq u_{1} \leq u_{2} \leq \ldots \leq u_{r} \leq v_{r} \leq \ldots \leq v_{2} \leq v_{1} \leq v_{0}$. Since $\mathbb{R}_{+}^{n}$ is regular, $u_{*}=\lim _{r \rightarrow \infty} u_{r}$ and $v_{*}=\lim _{r \rightarrow \infty} v_{r}$ are well defined and $u_{*} \leq v_{*}$. From the continuity of $\widehat{\mathbf{H}}$ it follows that $u_{*}=\widehat{\mathbf{H}}\left(u_{*}, v_{*}\right)$ and $v_{*}=\widehat{\mathbf{H}}\left(v_{*}, u_{*}\right)$, that is, $\left(u_{*}, v_{*}\right)$ is a coupled fixed point of $\mathbf{H}$. 
We now show that $\left(u_{*}, v_{*}\right)$ is minimal and maximal on $\left[0, v_{0}\right]$. Indeed, let $(\bar{u}, \bar{v})$ be another coupled fixed point of $\mathbf{H}$ on $\left[0, v_{0}\right]$. We have $0 \leq \bar{u} \leq v_{0}$ and $0 \leq \bar{v} \leq v_{0}$. Using the properties of $\widehat{\mathbf{H}}$ we obtain, by induction, $u_{r} \leq \bar{u} \leq v_{r}$ and $u_{r} \leq \bar{v} \leq v_{r}$ for all $r \in \mathbb{N}$, which implies (since $\mathbb{R}_{+}^{n}$ is closed) that $u_{*} \leq \bar{u} \leq v_{*}$ and $u_{*} \leq \bar{v} \leq v_{*}$, that is, $\left(u_{*}, v_{*}\right)$ is a minimal and maximal coupled fixed point of $\mathbf{H}$ on $\left[0, v_{0}\right]$.

Let $x_{*}$ be the least element solution of the problem $\operatorname{IGOCP}\left(\left\{\mathbf{T}_{k}\right\}_{k=1}^{m}, \mathbb{R}_{+}^{n}\right)$. To finish, it will be shown that $u_{*} \leq x_{*} \leq v_{*}$. First, we show that $0 \leq x_{*} \leq v_{*}$. We have $\mathbf{H}\left(\left[u_{*}, v_{*}\right]\right) \subseteq\left[u_{*}, v_{*}\right]$. Indeed, if $u_{*} \leq x \leq v_{*}$ then for every $r \in \mathbb{N}$, we have $u_{r} \leq x \leq v_{r}$. Since $\mathbf{H}(x)=\widehat{\mathbf{H}}(x, x)$ for every $x \in \mathbb{R}_{+}^{n}$, we obtain $u_{r+1} \leq \mathbf{H}(x) \leq v_{r+1}$ and since $\left[u_{r+1}, v_{r+1}\right] \subseteq\left[u_{r}, v_{r}\right]$ we have $\mathbf{H}(x) \in\left[u_{r}, v_{r}\right]$ for every $r \in \mathbb{N}$, that is, $\mathbf{H}\left(\left[u_{*}, v_{*}\right]\right) \subseteq\left[u_{*}, v_{*}\right]$. By Brouwer's fixed point theorem, $\mathbf{H}$ has a fixed point $\widehat{x}$. Obviously, $\widehat{x}$ is a solution of the problem $\operatorname{IGOCP}\left(\left\{\mathbf{T}_{k}\right\}_{k=1}^{m}, \mathbb{R}_{+}^{n}\right)$ and $u_{*} \leq \widehat{x} \leq v_{*}$. Since $x_{*}$ is the least element solution we have $0 \leq x_{*} \leq \widehat{x}$, which implies that $0 \leq x_{*} \leq v_{*}$. Now, since every fixed point of $\mathbf{H}$ is a coupled fixed point and $\left(u_{*}, v_{*}\right)$ is a minimal and maximal coupled fixed point of $\mathbf{H}$ on $\left[0, v_{0}\right]$ we must have $u_{*} \leq x_{*} \leq v_{*}$ and the theorem is proved.

Remarks. 1) The least element solution $x_{*}$ of the problem $\operatorname{IGOCP}\left(\left\{\mathbf{T}_{k}\right\}_{k=1}^{m}, \mathbb{R}_{+}^{n}\right)$, associated with a tolerant system $\mathbf{S}\left(\left\{f_{i j}^{k}\right\}\right)$ is independent of the tolerance $\Phi$.

2) An interesting situation when the operators $\mathbf{I}-\mathbf{T}_{k}(k=1, \ldots, m)$ are heterotonic is when $\mathbf{I}-\mathbf{T}_{k}=\mathbf{R}_{k}+\mathbf{P}_{k}$ with $\mathbf{R}_{k}$ isotone and $\mathbf{P}_{k}$ antitone. Indeed, in this case, let $\widehat{\mathbf{S}}_{k}(x, y)=\mathbf{R}_{k}(x)+\mathbf{P}_{k}(y)$ for all $(x, y) \in \mathbb{R}_{+}^{n} \times \mathbb{R}_{+}^{n}$.

6. On the construction of a tolerance. In the construction of a tolerance $\Phi$, an important requirement is that $(\mathbf{I}+\Phi)^{-1}$ is isotone. The following results can be used in this sense.

(I) We say that a matrix $\mathbf{A}=\left(a_{i j}\right)$ is an $\mathbf{M}$-matrix if $\mathbf{A}$ can be expressed in the form $\mathbf{A}=\alpha \mathbf{I}-\mathbf{B}$ with $\mathbf{B} \geq 0$ (i.e., $\mathbf{B}=\left(b_{i j}\right)$ and $b_{i j} \geq 0$ ) and $\alpha \geq \varrho(\mathbf{B})$, where $\varrho(\mathbf{B})$ is the spectral radius of $\mathbf{B}$. It is known that if $\mathbf{A}$ is an $\mathbf{M}$-matrix and $\mathbf{D}$ is a diagonal positive matrix, then $\mathbf{D}+\mathbf{A}$ is an $\mathbf{M}$ matrix. If $\mathbf{A}$ is an $\mathbf{M}$-matrix then $(\mathbf{I}+\mathbf{A})^{-1}$ is isotone. Hence, a candidate for a tolerance is $\Phi=\mathbf{A}$ if $\mathbf{A}$ is an $\mathbf{M}$-matrix.

(II) The same conclusion as in example (I) is true if $\Phi=\mathbf{A}$ and $\mathbf{A}$ is an $n \times n$ matrix such that $\mathbf{I}+\mathbf{A}$ satisfies the Hawkins-Simon condition (see: H. Nikaido, Convex Structures and Economic Theory, Academic Press, 1968, p. 90).

(III) Let $\psi=\left(\psi_{1}, \ldots, \psi_{n}\right)$ be a mapping from $\mathbb{R}^{n}$ into $\mathbb{R}^{n}$. We say that $\psi$ is a diagonal mapping if, for every $i=1, \ldots, n$, the component $\psi_{i}$ depends on $x_{i}$ only, where $x=\left(x_{1}, \ldots, x_{n}\right)$. If $\Phi: \mathbb{R}^{n} \rightarrow \mathbb{R}^{n}$ has a decomposition 
of the form $\Phi=\mathbf{A}+\psi$, where $\mathbf{A}$ is an $\mathbf{M}$-matrix and $\psi$ is a continuous diagonal increasing mapping, then $(\mathbf{I}+\Phi)^{-1}$ is isotone.

(IV) Let $\Phi: \mathbb{R}^{n} \rightarrow \mathbb{R}^{n}$ be a mapping such that $\Phi=\mathbf{A}+\psi$, where $\mathbf{A}=\left(a_{i j}\right)$ is an $n \times n$ matrix with $a_{i j}<0$ for all $i \neq j$ and $\psi=\left(\psi_{1}, \ldots, \psi_{n}\right)$ is a diagonal mapping. The following result is proved in [9]. If there exist $\alpha, \beta \in \mathbb{R}$ such that:

1) $\alpha+\beta>0$,

2) $\langle\mathbf{A} x, x\rangle \geq \alpha\langle x, x\rangle$ for all $x \in \mathbb{R}^{n}$,

3) $\left(\psi_{i}(s)-\psi_{i}(t)\right) /(s-t) \geq \beta$ for all $s, t \in \mathbb{R}, s \neq t$ and $i=1, \ldots, n$, then $(\mathbf{I}+\Phi)^{-1}$ is isotone and hence $\Phi$ is a candidate for a tolerance.

7. Estimation by global optimization. We also remark that the least element solution of the problem $\operatorname{IGOCP}\left(\left\{\mathbf{T}_{k}\right\}_{k=1}^{m}, \mathbb{R}_{+}^{n}\right)$ can be estimated by global optimization. If we consider on $\mathbb{R}^{n}$ the euclidean norm, then $\left(\mathbb{R}^{n},\langle\cdot, \cdot\rangle, \mathbb{R}_{+}^{n}\right)$ is a Hilbert lattice and the operations " $\wedge$ " and " $\vee$ " are continuous. If the mappings $\mathbf{T}_{k}(k=1, \ldots, m)$ are $\sigma$-Hölder continuous in the sense of definitions considered in [14], [17], [18], [29], we can show (see [14]) that $\mathbf{H}(x)$ is also $\sigma$-Hölder continuous (but generally with respect to another function $\sigma)$. If $y_{0}$ is a feasible element or $\left[0, v_{0}\right]$ is a strongly invariant segment for our problem, then by Theorem 2 (respectively Theorem 3 ) we obtain a conical segment $\left[0, v_{*}\right]$ (respectively $\left[u_{*}, v_{*}\right]$ ) containing the least element solution of our problem.

Hence we have to compute all the fixed points of $\mathbf{H}(x)$ on a conical segment, which is equivalent to computing all the global minima of the function $f(x)=\|x-\mathbf{H}(x)\|$ in a cube containing the conical segment where we have our solution.

But the function $f(x)$ is again $\sigma$-Hölder continuous function and its minimal value is zero. Thus, we can use the extension to $\sigma$-Hölder continuous functions of the cubic algorithm [12] or we can use the integral global optimization developed recently by Zheng [35], [36].

Probably, this last idea is a good motivation to extend to $\sigma$-Hölder continuous functions other methods developed in global optimization for Lipschitz functions.

We propose to work with $\sigma$-Hölder continuous functions, since this class is larger than the class of Lipschitz functions and for some numerical algorithms, as for example the cubic algorithm [7], we must know the value of the Lipschitz constant, which is not always easy to evaluate, while by imbedding theorems ([18], p. 361) we can know the function $\sigma$.

Conclusions. In this paper we presented two important ideas. First, the Implicit Generalized Order Complementarity Problem, which is a new subject in Complementarity Theory, can be used to construct and to study 
the extension of Leontief's system when the economic system works with several technologies. Second, we showed that the concept of tolerant system is a good model when the system is working with the functions $f_{i j}^{k}$ without the property of being increasing, as considered by other authors [2], [3], [30] etc. Third, we showed that a tolerant economic system, which is locally nonlinear and with the functions $f_{i j}^{k}$ not necessarily isotone, has a behavior similar to a classical Leontief model.

\section{References}

[1] Y. M. Bershchanskiǔ and M. V. Meerov, The complementarity problem: theory and methods of solution, Automat. Remote Control 44 Part I (1983), 687-710.

[2] P. Bod, On closed sets having a least element, in: Lecture Notes in Econom. and Math. Systems 177, Springer, 1976, 23-34.

[3] —, Sur un modèle non-linéaire de rapports interindustriels, RAIRO Rech. Opér. 11 (1977), 405-415.

[4] J. M. Borwein and M. A. H. Dempster, The linear order complementarity problem, Math. Oper. Res. 14 (1989), 534-558.

[5] R. W. Cottle, J. S. Pang and R. E. Stone, The Linear Complementarity Problem, Academic Press, 1992.

[6] A. Ebiefung and M. Kostreva, The generalized Leontief input-output model and its application to the choice of new technology, Ann. Oper. Res. 44 (1993), 161-172.

[7] E. A. Galperin, The cubic algorithm, J. Math. Anal. Appl. 112 (1985), 635-640.

[8] C. R. Glassey, A quadratic network optimization model for equilibrium simple commodity trade flow, Mat. Programming 14 (1978), 98-107.

[9] K. Glashoff and B. Werner, Inverse monotonicity of monotone L-operator with applications to quasilinear and free boundary value problems, J. Math. Anal. Appl. 72 (1979), 89-105.

[10] D. Guo and V. Lakshmikantham, Coupled fixed points of nonlinear operators with applications, Nonlinear Anal. 11 (1987), 623-632.

[11] G. Is ac, Problèmes de complementarité (en dimension infinie), Publ. Dépt. Math. Univ. Limoges, 1985.

[12] -, Complementarity problem and coincidence equations on convex cones, Boll. Un. Mat. Ital. B 6 (1986), 925-943.

[13] —, Complementarity Problems, Lecture Notes in Math. 1528, Springer, 1992.

[14] - Iterative methods for the general order complementarity problem, in: Approximation Theory, Spline Functions and Applications, S. P. Singh (ed.), Kluwer Acad. Publ., 1992, 365-380.

[15] G. Isac and M. Kostreva, The generalized order complementarity problem, J. Optim. Theory Appl. 71 (1991), 517-534.

[16] - , - Kneser's theorem and the multivalued generalized order complementarity problem, Appl. Math. Lett. 4 (6) (1991), 81-85.

[17] L. G. Khanin, Kantorovich-Rubinshtein duality for Lipschitz spaces defined by differences of arbitrary order, Soviet Math. Dokl. 42 (1991), 220-224.

[18] A. Kufner, O. John and S. Fučík, Function Spaces, Noordhoff, 1977.

[19] J. Łoś and M. W. Łoś (eds.), Mathematical Models in Economics, PWN and NorthHolland, 1974. 
[20] J. Łoś and M. W. Łoś (eds.), Computing Equilibria: How and Why?, PWN and North-Holland, 1978.

[21] L. Mathiesen, Computational experience in solving equilibrium models by a sequence of linear complementarity problems, Oper. Res. 33 (1985), 1225-1250.

[22] V. I. Opoŭtsev, A generalization of the theory of monotone and concave operators, Trans. Moscow Math. Soc. 2 (1979), 243-279.

[23] J. S. Pang, I. Kaneko and W. P. Hallman, On the solution of some (parametric) linear complementarity problems with applications to portfolio selection, structural engineering and actuarial graduation, Math. Programming 16 (1979), 325-347.

[24] J. S. Pang and P. S. C. Lee, A parametric linear complementarity technique for the computation of equilibrium prices in a single commodity spatial model, ibid. 20 (1981), 81-102.

[25] O. Paris, Revenue and cost uncertainty generalized mean-variance and the linear complementarity problem, Amer. J. Agricultural Econom. 61 (1979), 268-275.

[26] A. L. Peressini, Ordered Topological Vector Spaces, Harper \& Row, 1967.

[27] E. L. Peterson, The conical duality and complementarity of price and quality for multicommodity spatial and temporal network allocation problem, Discussion paper 207, Center for Mathematical Studies in Economics and Management Science, Northwestern Univ., 1976.

[28] M. H. Schneider, Single-commodity spatial equilibria: a network complementarity approach, Ph.D. Thesis, Dept. Industrial Engineering and Management Sciences, Northwestern Univ., Evanston, Ill., 1984.

[29] F. Schulz, Regularity Theory for Quasilinear Elliptic Systems and Monge-Ampère Equations in Two Dimensions, Lecture Notes in Math. 1445, Springer, Berlin, 1990.

[30] A. Tamir, Minimality and complementarity properties associated with Z-functions and M-functions, Math. Programming 7 (1974), 17-31.

[31] J. von Neumann, A model of general economic equilibrium, Rev. Econom. Stud. $13(1945 / 1946), 1-9$.

[32] R. Wilson, Bilinear complementarity problem and competitive equilibria of piecewise linear economic model, Econometrica 46 (1978), 87-103.

[33] G. Wintgen, Indifferente Optimierungs Problem, Beitrag zur Internationalen Tagung, Mathematik und Kybernetik in der Ökonomie, Berlin, 1964, Konferenzprotokoll, Teil II, Akademie-Verlag, Berlin, 3-6.

[34] J. C. Yao, A basic theorem of complementarity for the generalized variational-like inequality problem, J. Math. Anal. Appl. 158 (1991), 124-138; The generalized quasi-variational inequality problem with applications, ibid., 139-160.

[35] Q. Zheng, Optimality conditions for global optimization (I) and (II), Acta Math. Appl. Sinica 1 (2, 3) (1985), 66-78 and 118-132.

[36] - Robust analysis and global optimization, Internat. J. Computers Math. Appl. $21(6 / 7)(1991), 17-24$.

G. Isac

Department of Mathematics

Royal Military College of Canada

Kingston, Ontario

Canada, K7K 5L0
M. Kostreva

Department of Mathematical Sciences Clemson University Clemson, South Carolina 29634-1907

U.S.A. 
